\title{
A Novel Concept of Correcting Presbyopia: First Clinical Results with a Phakic Diffractive Intraocular Lens
}

This article was published in the following Dove Press journal: Clinical Ophthalmology

\section{Ruediger Schmid (D) \\ Holger Luedtke}

Accuratis. Private Practice for Refractive Surgery, Ulm, Germany
Correspondence: Ruediger Schmid Tel +49 731 28065680

Email r-schmid@gmx.de
Purpose: To evaluate the effect of a novel technique to correct presbyopia. A phakic IOL (presbyopic IPCL; implantable phakic contact lens) with a diffractive optic is implanted and its impact on visual acuity, refraction, patient satisfaction in patients striving for spectacleindependence is evaluated.

Design: Retrospective noncomparative open-label clinical trial.

Methods: Sixteen eyes of 8 patients (average age 47 years) had a presbyopic IPCL implanted in the posterior chamber. The visual acuity on different distances, refractive status, corneal topography, endothelial cell density, anterior chamber depth, white-to-white, mesopic pupil size and intraocular pressure (IOP) were measured before implantation of this novel phakic IOL with diffractive optic and four weeks after surgery.

Results: At follow-up four weeks after surgery, 9 of the 16 eyes were emmetropic and uncorrected distance visual acuity was at least 0.8 . Near vision was excellent in all patients without the need to wear reading glasses. There was neither a significant change in IOP nor a significant surgical impact on endothelial cells. Patient satisfaction was high. There was no major complaint of halos or glare.

Conclusion: The presbyopic IPCL can provide the presbyopic patient with good visual acuity and spectacle-independence for far and near distance. We found this novel technique to have a good safety profile during the surgical procedure and our short follow-up period. Further long-term follow-up is mandatory.

Keywords: refractive surgery, myopia, phakic intraocular lens, pIOL, presbyopia, IPCL

\section{Introduction}

Presbyopia is one of the most common refractive problems, currently affecting approximately 1.8 billion people worldwide with an increase to 2.1 billion expected by the year $2030 .{ }^{1}$ It also is the most common refractive disorder in people aged 40 and older. A number of surgical procedures have been introduced of which none has become dominant; presbyopia correction has been repeatedly described as the proverbial last frontier in refractive surgery. ${ }^{2}$ Among the methods used to correct presbyopia surgically are interventions to induce monovision and corneal inlays like the KAMRA (Acufocus, Inc.) or the Flexivue Microlens (Presbia, Inc.). There are aberrations-based presbyopic LASIK, attempts to steepen the cornea by a femtosecond laser treatment known as Intracor, ${ }^{3}$ and scleral procedures.

Finally, there is the option of implanting an intraocular lens (IOL) by removal of the crystalline lens, either by the way of cataract surgery or specifically (for 
a younger patient) to provide spectacle-independence. Multifocal $\mathrm{IOLs}^{4-6}$ and accommodating IOLs are implanted for that purpose although the range of natural accommodation cannot be restored by the latter. Patients with multifocal IOLs tend to experience glare, halos and some decrease in contrast sensitivity. Extended-Depth-ofFocus-IOLs will produce less halos yet can`t provide complete spectacle-independence for near vision. ${ }^{7}$

A phakic intraocular lens (pIOL) is a supplementary lens that is inserted into the anterior or posterior chamber of the eye to correct refractive errors. Unlike in cataract surgery or refractive lens exchange, the patient's crystalline lens is not removed in a purely refractive procedure. There are a number of indications for the implantation of pIOLs, ranging from hyperopia, myopia and astigmatism to special situations like keratoconus, following a corneal transplant and to correct residual refractive errors in pseudophakia. ${ }^{8}$

Recently, a new phakic posterior-chamber diffractive IOL for presbyopia correction has been introduced (presbyopic IPCL). In our surgical center, we started implanting this pIOL in April 2017 in presbyopic patients motivated by the desire to become spectacle-independent. To the best of our knowledge, this is the first clinical report on refractive and visual results as well as, certainly limited due to the short follow-up period, a first impression of the safety of this novel device. This novel presbyopic IPCL represents an overdue innovation combining the established procedure of inserting a phakic lens with the established method of achieving a range of vision from distance to near by diffractive optics.

Correcting presbyopia with a phakic lens could be advantageous to refractive lens exchange by avoiding potential retinal alteration like retinal tears or macular edema or macular hole when the vitreous is still attached at the time of surgery.

\section{Methods}

\section{Study Design}

This retrospective noncomparative open-label clinical trial was performed at the accuratis Eye Clinic in Ulm, Germany. The study was performed in accordance with the tenets of the Declaration of Helsinki; local ethical committee LAEK Baden-Württemberg. Patients were screened for eligibility, and informed consent and consent to publish was obtained from all eligible patients. Sample Size was 16 healthy eyes of 8 patients.

\section{Presbyopic IPCL as a Posterior-Chamber Phakic Intraocular Lens}

The presbyopic IPCL (Care Group India, Gujarat, India) is a recently developed implantable phakic refractive IOL with a diffractive optic (Figure 1). Its indication is the correction of presbyopia in patients between approximately 40 and 60 years of age, that is before the onset of cataract. The IPCL is also implanted to correct myopia, hyperopia and astigmatism in addition to presbyopia. ${ }^{8}$

The presbyopic IPCL is made of hydrophilic hybrid acrylic material and has a diopter range between -0.5 and -30.0 diopters (D) for myopia as well as +0.5 to $+6.0 \mathrm{D}$ to correct hyperopia. Cylinder range is +1.0 to $+8.0 \mathrm{D}$; there is a customized cylinder axis. For implantation, an incision of $2.8 \mathrm{~mm}$ size is required. Anterior chamber depth must be at least $2.8 \mathrm{~mm}$. IPCL for myopia have a central opening to allow unrestricted aqueous flow; in these eyes an iridectomy is not necessary. The lens has four additional

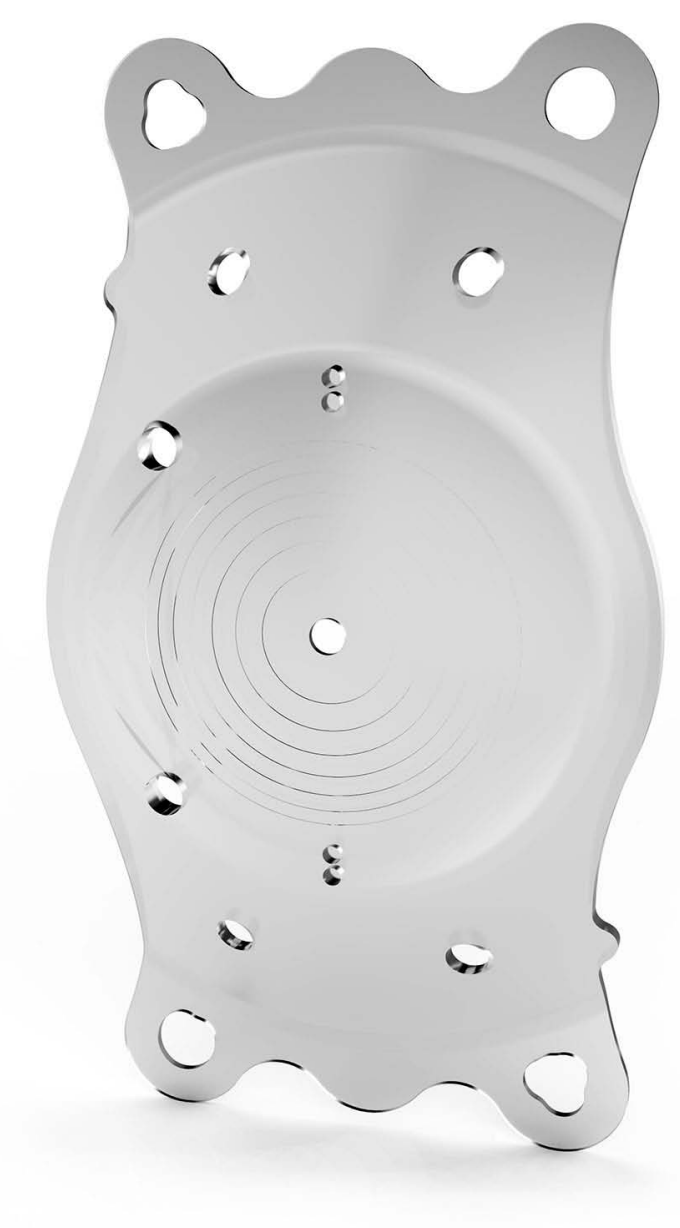

Figure I Presbyopic IPCL lens. 
holes in the periphery of its optic zone to allow aqueous flow. The IPCL has a diffractive optical zone of $5.8 \mathrm{~mm}$ and is manufactured with near additions between $+1.5 \mathrm{D}$ and +4.0 D. Optical diameters of the ICPL can be customized to accommodate for large pupils. Overall diameter of the IPCL ranges from 11.00 to $14.00 \mathrm{~mm}$ in steps of $0.25 \mathrm{~mm}$.

\section{Eligibility Criteria}

The study comprised 16 eyes of 8 healthy patients that received a presbyopic IPCL to correct their presbyopia only or together with myopia or astigmatism. Patients' age had to be between 40 and 60 years at the time of implantation. Corrected distance visual acuity had to be 1.0 decimal or better in each eye. Ocular pathologies like glaucoma, uveitis, corneal opacities, cataract or significant posterior segment abnormalities were considered exclusion criteria. The same applied to any relevant systemic affection. Patients had no other medication but mild antihypertensives in two cases.

\section{Preoperative Examination and Lens Calculation}

Decimal visual acuity was measured with the Early Treatment Diabetic Retinopathy Study (ETDRS) chart (Precision Vision) at 5 meters and with Birkhaeuser Charts at $40 \mathrm{~cm}$. Monocular and binocular uncorrected distance visual acuity (UDVA) at 5 meters and near visual acuity (UNVA) at $40 \mathrm{~cm}$ as well as corrected distance (CDVA) and distance-corrected near (DCNVA) visual acuity were measured. The (remaining) accommodative power of the natural crystalline lens was quantified which was essential in determining the additive power of the IPCL. Near correction of the IPCL was chosen to be $0.5 \mathrm{D}$ additive to the patient's reading glasses (median of near addition of IPCL was $2 \mathrm{D}$, quartiles 1.75 and 2.19). Subjective and objective refraction in miosis and cycloplegia was determined. The power of the IPCL was chosen according to subjective refraction, taking into account the position of the phakic lens in relation to corneal vertex distance. A toric IPCL was selected when astigmatism was $1 \mathrm{D}$ or more. A general slit-lamp examination was performed, of particular importance were the anterior chamber depth and the status of the natural lens. Specular microscopy of the corneal endothelium was performed to ensure endothelial cell density to exceed 2000 cells/qmm (EM3000, Tomey). Computerized keratometry, corneal topography, measurement of internal anterior chamber and white-to white were performed, pupil size was measured under low mesopic conditions with the Sirius device (bon optic) at 0.04 lux. Intraocular pressure (IOP) was measured by applanation tonometry. Macular pathologies were excluded by optical coherence tomography (3D OCT 2000 , Topcon).

\section{Surgical Technique}

All surgeries were performed by one experienced surgeon (R.S.), both eyes on the same day in a separate surgery.

All surgeries were done under propofol and larynx mask. The eye and lid were washed by Povidone-Iodine solution. A $2.8 \mathrm{~mm}$ incision was performed, paracenteses were added at 10 and 2 o'clock. As ophthalmic viscosurgical device (OVD), HPMC was injected in the anterior chamber and the shooter system was carefully inserted. The IPCL was gently injected bimanually, i.e. the opening of the lens inside the eye was controlled by a Sinsky hook. The edges of the lens were cautiously implanted under the iris and the lens was stabilized in a horizontal position. Care was taken to neither touch the corneal endothelium nor the natural lens during the implantation process. If the patient had a subjective astigmatism of $1 \mathrm{D}$ or more, the toric version of the presbyopic IPCL was implanted. At the end of the procedure, OVD was carefully removed. Application of acetylcholine (Miochol E) proved generally not to be necessary. After surgery, dexamethasone-gentamycin ointment was applied and a transparent monoculus was fixed on each eye. Postoperatively, $500 \mathrm{mg}$ acetazolamide was given, topically the patients received prednisolone $1 \%$ eye drops for 10 days and moxifloxacin eye drops for 5 days, each 5 times a day.

\section{Postoperative Examination}

Four weeks after implantation, refractive status, UDVA, UNVA, CDVA and DCNVA were evaluated. The anterior and posterior chamber anatomy and the situation of the implanted pIOL were visualized by Scheimpflug imaging using the Sirius device. During slit-lamp examination, special care was taken to find evidence of, or rule out, signs of inflammation, pigment dispersion and other forms of intraocular irritation. The endothelial cell density was measured by specular microscopy. IOP was measured by applanation tonometry. Overall patient satisfaction concerning quality of vision was inquired and rated on a scale from 1 (poor) to 10 (excellent). The patients were asked how satisfied they were with the visual acuity on different distances (driving, computer, reading), with the comfort of the procedure and if they suffered from halos and glare (demonstrated by simulation pictures).

In this study, emphasis was placed on safety of the implantation procedure and the efficacy of the new IPCL. 
Thus, photic phenomena typical for diffractive optics were not specifically evaluated nor analyzed.

\section{Statistical Analysis}

A Wilcoxon signed rank test with continuity correction was used to evaluate the pre and post values: Spherical equivalent of refraction, UDVA, UNVA, astigmatism, intraocular pressure, endothelial cell count. The significance level was set to 0.05 . For IPCL vaulting respectively patients' overall satisfaction, the single values were analyzed as median and quartile.

\section{Results}

\section{Preoperative Findings}

Our patients were 42 to 57 years old (median 45.5, quartiles $44,50.25)$ at the time of implantation, $25 \%$ were female. Myopia ranged from $-1.75 \mathrm{D}$ to $-11.25 \mathrm{D}$ (median $-3.5 \mathrm{D}$, quartiles $-7.25 \mathrm{D}$ and $-2.06 \mathrm{D}$ ), astigmatism from $0 \mathrm{D}$ to 1.75 $\mathrm{D}$ (median $-0.75 \mathrm{D}$, quartiles $-1.0 \mathrm{D}$ and $-0.5 \mathrm{D}$ ). None of our eyes was hyperopic, one eye was emmetropic. Internal anterior chamber depth was $3.34 \mathrm{~mm}$ in mean (3.04-3.6), mesopic pupil size was $5.26 \mathrm{~mm}$ in mean (4.5-6.3).

\section{Refraction and Visual Acuity}

Four weeks after surgery with the IPCL, 9 of the 16 eyes were emmetropic, the rest had myopia between -0.25 and -1.0 D (Figure 2; Wilcoxon pre/post $p<0.001$ ). Mean of spherical equivalent after surgery was $0 \mathrm{D}$ (quartiles $-0.25 \mathrm{D}, 0 \mathrm{D})$ with 2 eyes more than $0.5 \mathrm{D}(13 \%)$.

Astigmatism after surgery was below or equal $0.5 \mathrm{D}$ in 15 of 16 eyes, partly corrected by toric IPCL, with only 1 eye left with $1 \mathrm{D}$ astigmatism (Figure 3; Wilcoxon pre/post $\mathrm{p}<0.005$ ).

Median of best UDVA was 0.1 (quartiles $0.02,0.3$ ) before surgery, the maximum being 1.0 due to the one emmetropic eye. Postoperatively, median of UDVA was 1.0 (quartiles 0.9 , 1.0, maximum 1.2, minimum 0.8). UNVA which preoperatively was good in our myopic population (median 1.0, quartiles 1.0,1.2), postoperatively was in median 1.0 (quartiles $0.9,1.0$, maximum 1.2 , minimum 0.63 ). UDVA and UNVA after surgery are shown in Figure 4. Intermediate visual acuity was not analyzed separately. CDVA was not significantly different before and after surgery in our cases with clear crystalline lens. DCNVA was not compared before and after surgery because specifically, the poor initial DCNVA was a key request for surgery in our presbyopic population.

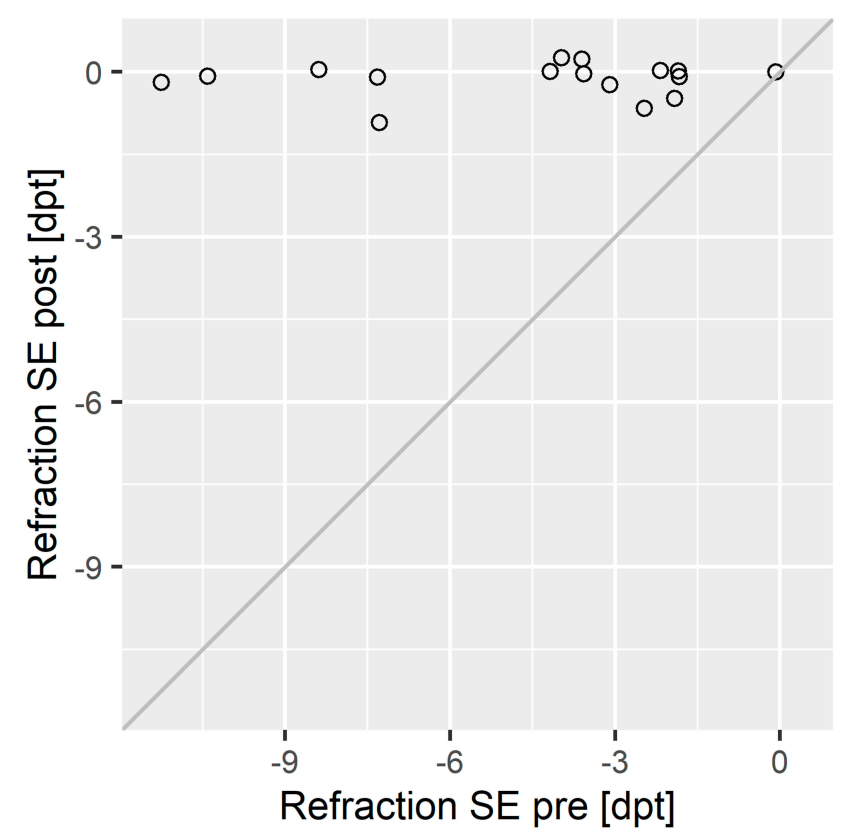

Figure 2 Spherical equivalent (SE) of refraction in diopters before and after surgery.

Defocus curves for a 43 years old patient with a $1.5 \mathrm{D}$ near addition and a 48 years old patient with a $2.5 \mathrm{D}$ near addition are shown in Figure 5. Whereas the 43 years old achieved a continuous level of visual acuity from far to near, for the 48 years old, the intermediate visual acuity dropped down significantly.

\section{Safety, Complications and Patient Satisfaction}

There was no significant change in IOP (Figure 6; Wilcoxon $\mathrm{p}=0.5)$, the average was preoperatively $13.8 \mathrm{~mm} \mathrm{Hg}(12-16 \mathrm{~mm} \mathrm{Hg})$; postoperatively the average IOP was $14.0 \mathrm{~mm} \mathrm{Hg}(12-17 \mathrm{~mm} \mathrm{Hg})$.

There was no significant loss of corneal endothelial cell density (Wilcoxon $=0.05$ ) which preoperatively was measured to be on average 2475 cells per $\mathrm{mm}^{2}$ (2333-2898), postoperatively it was 2441 cells per $\mathrm{mm}^{2}$ (2314-2862). The median vault of the IPCL was $0.28 \mathrm{~mm}$ (quartiles $0.16,0.38$, maximum 0.9 , minimum 0.1 ; with 4 eyes below $0.2 \mathrm{~mm}$ ) and in all cases in sufficiently good relation to anterior chamber depth, see Figure 7.

In 1 case, there was a significant rotation of the toric IPCL that required a reposition a few days later. In 1 case, we had an upside-down implantation of the IPCL which was corrected in the same surgery.

Overall patient satisfaction concerning quality of vision was high. The average score of each eye's individual outcome was $8.7(6-10)$ on a scale from 0 to 10 . 


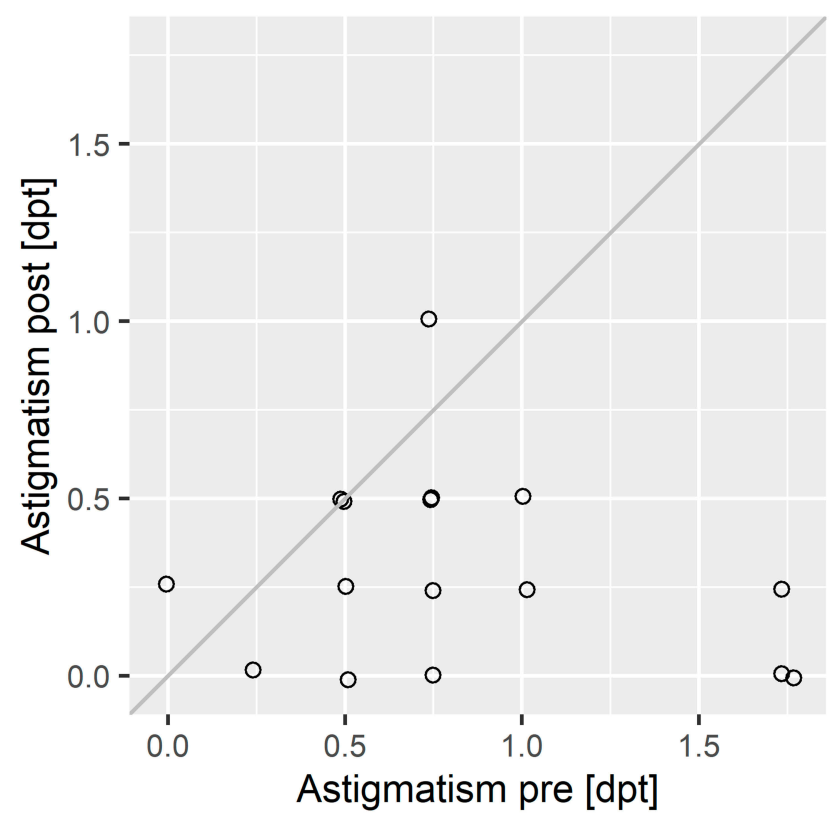

Figure 3 Astigmatism (diopters) before and after surgery.

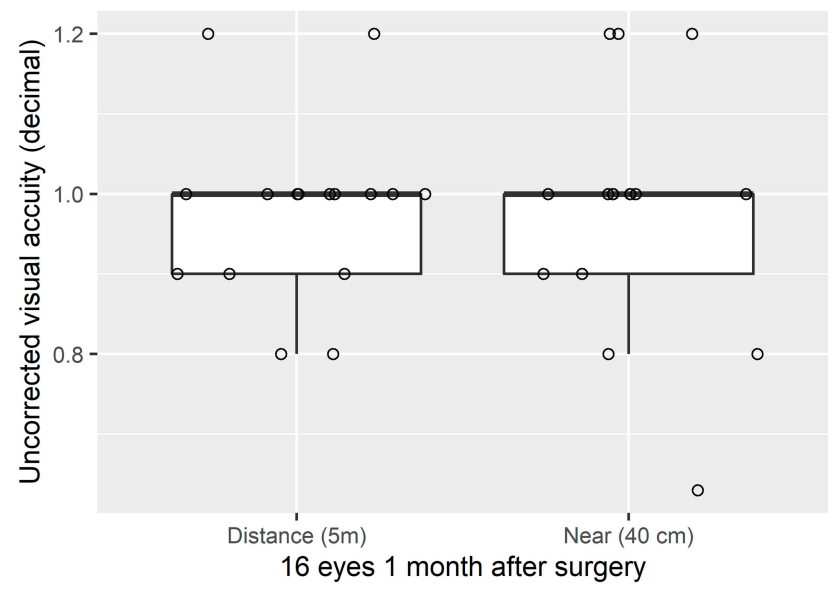

Figure 4 Uncorrected distance visual acuity (UDVA) and uncorrected near visual acuity (UNVA) after surgery for each single eye (decimal).

\section{Discussion}

Phakic IOL (pIOL) are an established procedure in the treatment of high myopia and widely considered safe and predictable. ${ }^{9}$ PIOL are used also in hyperopia and astigmatism. In high myopia, the quality of vision with a pIOL has been described as superior to Lasik. ${ }^{10,11}$

Among the advantages of pIOL are the relatively fast visual recovery and the good refractive stability as well as the reversibility should complications occur or the patient is not satisfied with the results. ${ }^{12}$ There are three different pIOL designs with respect to the implantation site: the (obsolete) angle-supported anterior chamber lens, the iris- claw anterior chamber lens, and the posterior chamber lens. The main concern with the anterior chamber pIOL is the prospect of potential loss of corneal endothelial cells due to the proximity of the implantation site. ${ }^{13,14}$ However, there seems to be a stabilization with time. ${ }^{15}$

Posterior chamber phakic IOLs come with a lesser risk of endothelial cell loss. A recent study by Shimizu et al documented an endothelial cell loss of just $0.5 \%$ over a 5 -year period ${ }^{16}$ after implantation of a modern posterior chamber pIOL. A major concern with this design has been the potential rise in IOP and the possible contribution to cataract formation. In the study by Shimizu et al with 64 eyes that received two different types of posterior chamber pIOL, no significant increase in IOP $(>21 \mathrm{~mm} \mathrm{Hg})$ occurred in any case during the 5-year observation period. Since refractive patients tend to be in general younger than cataract patients, long-term safety and stability is essential. A mean follow-up of 47 months in a group of 349 eyes in 216 patients with a mean age of 29 years, as reported by Gomez-Bastar et al with a complication rate of about $2 \%$ seems reasonable but it is still a limited time period; ${ }^{17}$ relatively young patients undergoing posterior chamber pIOL implantation will most likely wear these lenses for decades before reaching the age of cataract surgery and thus a possible revision of their IOL status.

Presbyopia correction has initially not been considered by the designers and manufacturers of current pIOLs and it was just in 2015 that results of an experimental study with a potential pIOL design for (relatively) young presbyopic patients were published. ${ }^{18}$ The innovation of the presbyopic IPCL is to combine a diffractive optic with a hydrophilic acrylic posterior chamber IOL. The IPCL is manufactured in India and has been implanted in Asia and in a few clinics in Europe like, for instance, in France or Spain.

In our case series, implantation of the presbyopic IPCL turned out to be in no way more surgically challenging than any other intervention with pIOLs. The shooter system provided by the manufacturer was relatively easy to handle and loading the IOL into the cartridge went smoothly due to the latter's thin but slightly more rigid material compared to collamer. The implantation process with this device is, however, not without challenges. There is a chance that the lens turns inadvertently from posterior to anterior during implantation. The edges of the haptic may come out of the cartridge turned upside, so care must be taken to prevent any contact with the corneal endothelium in 


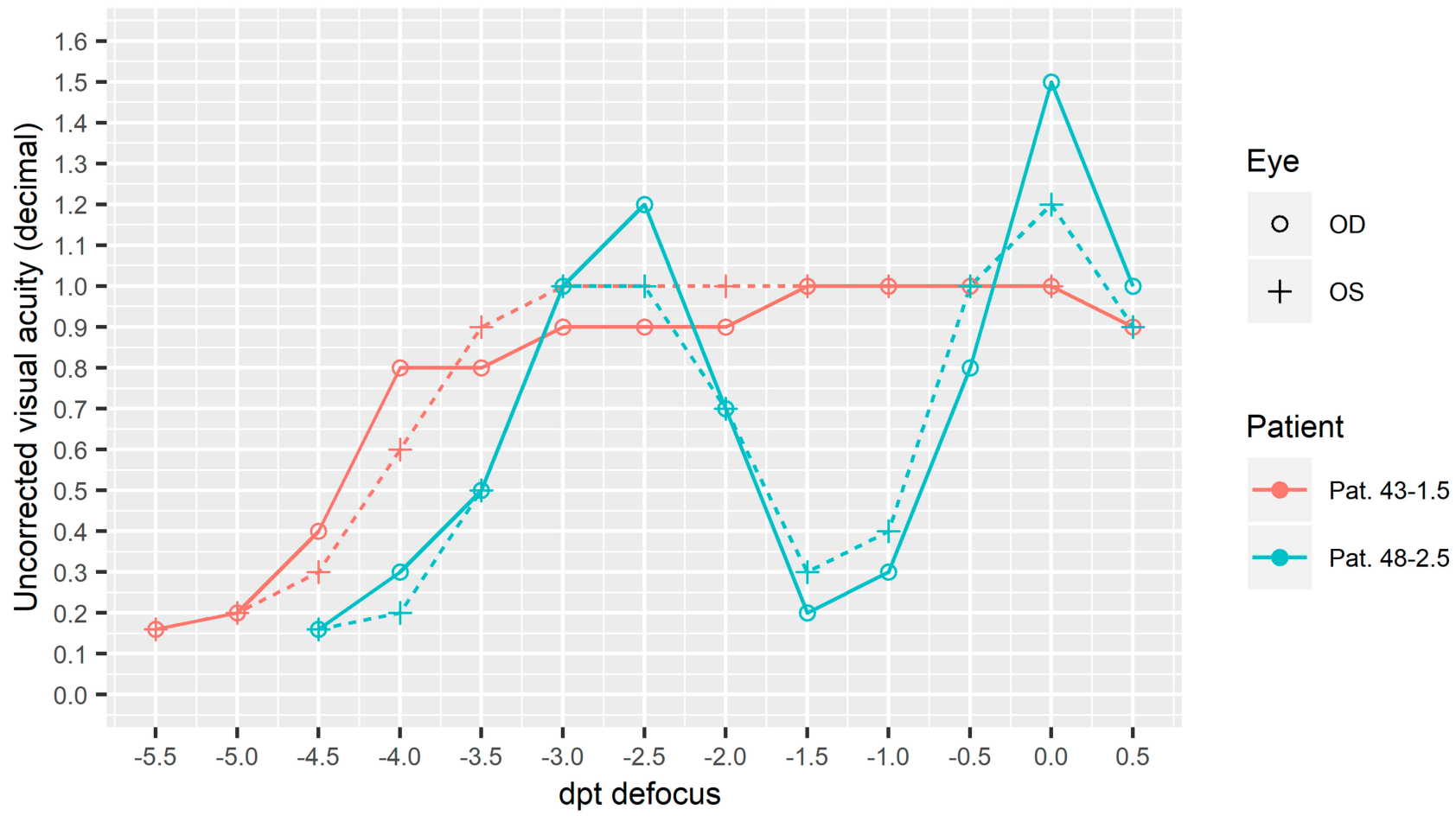

Figure 5 Monocular defocus curves for two patients' right and left eye with the presbyopic IPCL. Red= 43 ys, I.5 D near add, blue= 48 ys, $2.5 \mathrm{D}$ near add.

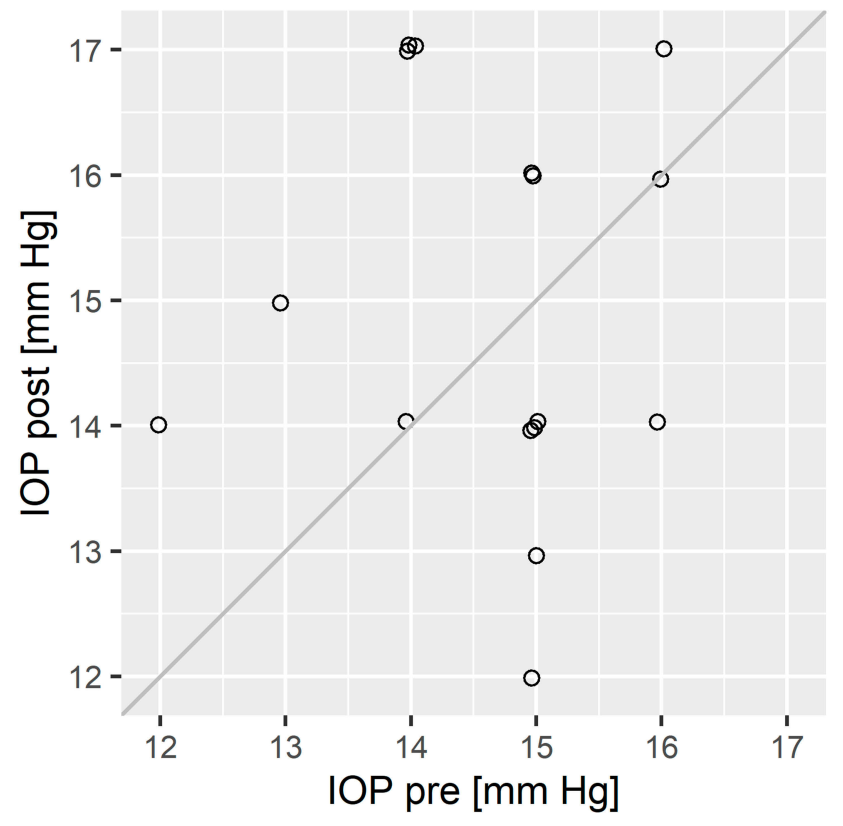

Figure 6 Intraocular pressure (IOP, in $\mathrm{mmHg}$ ) before and after surgery.

this situation. Changing the implantation technique so that the IOL is inserted into the cartridge in the same convexity as it is implanted in the eye could result in touching the crystalline lens. Otherwise, there were no surgical problems or intraoperative complications.

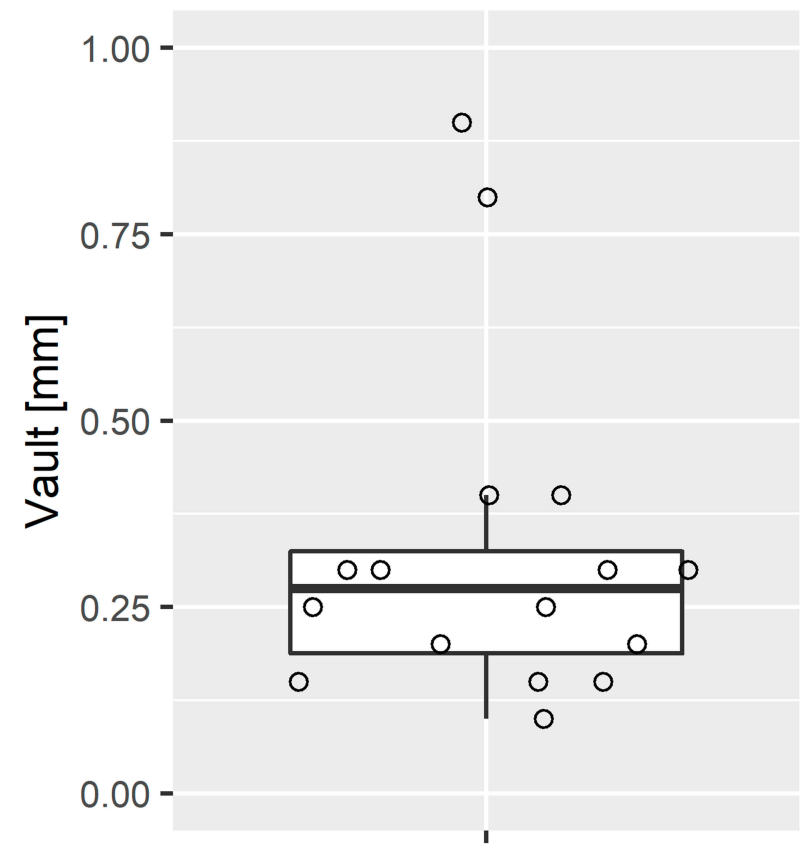

16 eyes 1 month after surgery

Figure 7 Vaulting of the IPCL (space between crystalline lens and IPCL) in $\mathrm{mm}$ for each single eye.

The refractive results were convincing. Most eyes which have with one exception been myopic before 
surgery, were emmetropic four weeks postoperatively. Myopia more than $0.5 \mathrm{D}$ in 2 eyes might be due to poor lens power calculation. Near binocular visual acuity without glasses was 1.0 or better in 7 of our 8 patients. In our group, none of the patients needed to wear glasses anymore, except on rare occasions like weak reading light. The quality of near vision in the long-term follow-up will undergo some minor change because we adjusted the power of the near segment to the age of the patient: Usually, a near addition of $0.5 \mathrm{D}$ higher than the patient's reading glasses was chosen. However, even with further decline of residual accommodation of the crystalline lens, there will always remain some extended depth of vision.

As assumed, in our younger patients, the defocus curve showed a good visual acuity over a broad range from far to near, presumably because of considerable residual accommodation. In our older patients with less residual accommodation, the presbyopic IPCL behaved like a bifocal lens in some cases, however without any patient's complaint about poor intermediate vision.

Overall patient satisfaction regarding the quality of vision was high. There were only some minor complaints about straylight when driving at night. This is interesting, for the natural crystalline lens could potentially increase straylight of the added diffractive optic.

During our short follow-up period, we did not see major complications in our patients. There were no signs of early endothelial cell damage, but endothelial cell loss due to apoptosis would naturally show up later in the long term. We did not observe any new opacification of the natural lens. If there was any rise in IOP, it was mild and never reached values that had to be treated. Vaulting was high in two eyes $(0.9$ and $0.8 \mathrm{~mm})$ with also sufficiently deep anterior chamber (internal anterior chamber depth $3.23 \mathrm{~mm}$ and $3.04 \mathrm{~mm}$ ), thus still a large enough gap between IPCL and cornea. We could leave the IPCL in these eyes, however, sizing of the IPCL was too large in these cases. Generally, sizing of the IPCL is very accurate due to the sizing steps of $0.25 \mathrm{~mm}$. Calculation of IPCL size is done by Care Group.

With the multifocal optic located on the anterior surface of the IPCL, long-term abrasion of iris pigment cannot completely be ruled out. We could not observe any significant surgically induced pigment dispersion. There were no signs of damage to the zonulae.

Regarding the potential safety profile of this new type of intraocular lens, the results of two studies with another, slightly older version of the IPCL might give an impression of what to expect. Vasavada et al reported the efficacy and safety of a monofocal IPCL - whose design and mode of implantation are comparable to the subject of our investigation - to correct high myopia (preoperatively on average $-16.5 \mathrm{D}$ ) in a group of patients just a bit larger than ours. The refractive efficacy was good, the average postoperative refractive error was $-0.89 \mathrm{D}$, providing a mean UDVA of $0.38 \log$ MAR. After a follow-up of 3 years, not a single eye lost any line of visual acuity. One eye $(3.3 \%)$ developed anterior subcapsular cataract but did not require cataract surgery at 3 years of follow-up. Two eyes $(6.6 \%)$ developed a mild transient increase in intraocular pressure, which required topical medication only for 3 months. The percentage of endothelial cell loss at 3 years was $9.73 \%$. The mean postoperative IOP in this group was not significantly different from preoperatively at any of the follow-up visits over the 3 years. ${ }^{19}$ In an even newer publication, Sachdev et al investigated the longterm safety of the monofocal IPCL that war implanted in 134 eyes to correct myopia (preoperatively on average -9.45 D). Postoperative examinations took place at 1, 6 and 12 months after the implantation. There were no intraoperative complications. Postoperatively, the most common complication was cataract formation (the patients' average age at the time of the intervention was 25 years and therefore way before cataract age) in 3 eyes $(2.2 \%)$ of which one eye required cataract surgery. Four eyes developed transient increased IOP due to steroid response ( 3 eyes) and pupillary block glaucoma (1 eye). The endothelial cell loss noted over a 1-year follow-up was $2.01 \%$. The authors concluded IPCL to be a safe and effective treatment modality. ${ }^{20}$

We did not find any indication that safety will become a major problem after implantation of the presbyopic IPCL. In one case in our group of patients, an explantation and re-implantation during the primary surgical procedure was necessary because the IOL turned out to be implanted upside-down due to the shooting process. While implantation went smoothly in most cases, there seems to still be room for improvement of the shooter device.

The position of the toric presbyopic IPCL (usually implanted in patients with an astigmatism of $1 \mathrm{D}$ and higher) was sufficiently stable. There was one revision because of a significant rotation that affected visual comfort. It may be an advantage that sizing of the IPCL is subtle graded and that the toric IPCL is always positioned horizontally due to its customized cylinder. That means, the sizing will usually fit very well because the white-to- 
white is measured horizontally, too. However, the haptics of the IPCL are very thin and obviously could slide into the sulcus.

There is still no world-wide standard protocol to report the refractive outcomes of presbyopia surgery; creating such a protocol will become increasingly necessary with new procedures being introduced and longer follow-ups of patients with the currently existing procedures will be published. $^{21}$ The results of our case series are encouraging. It should be noted that in most cases, we did not find proof for a real panfocality that one could imagine as the IPCL's optic principle because the natural lens still can accommodate. There were rather spontaneous levels of focality like in trifocal IOLs after lens removal. However, there is a wide depth of focus for near vision and almost no transitional zone between near and intermediate vision.

Our study group is relatively small, and the follow-up period is quite limited. It is, however, to the best of our knowledge, the first of its kind. The focus was on the visual results of this unique diffractive concept which can be assessed after a limited postoperative time period while for a valid safety profile a follow-up of a couple of years would be required - for that it is given the novelty of the device, currently too early. The results are encouraging and should be supported by further investigations with a larger number of patients and longer observations periods. We will track the patients of this study for the next years.

In conclusion, the visual and refractive results after implanting the presbyopic IPCL were good, rendering the procedure which aims primarily at spectacle-independence very successful. Patient satisfaction was high as was independence from wearing glasses. The reversibility of the procedure is a strong psychological factor for the patients and is a supporting factor for deciding in favor of this option. Given the increasing number of presbyopes, there is a huge potential demand for an innovation like the presbyopic IPCL.

\section{Disclosure}

The authors report no conflicts of interest in this work.

\section{References}

1. Fricke TR, Tahhan N, Resnikoff S, et al. Global prevalence of presbyopia and vision impairment from uncorrected presbyopia: systematic review, meta-analysis, and modelling. Ophthalmology. 2018;125 (10):1492-1499. doi:10.1016/j.ophtha.2018.04.013

2. Alio JL, Alio Del Barrio JL, Vega-Estrada A. Accommodative intraocular lenses: where are we and where we are going. Eye Vis (Lond). 2017;4(1):16. doi:10.1186/s40662-017-0077-7
3. Holzer MP, Knorz MC, Tomalla M, et al. Intrastromal femtosecond laser presbyopia correction: 1-year results of a multicenter study. J Refract Surg. 2012;28(3):182-188. doi:10.3928/1081597X-20120203-01

4. Mojzis P, Kukuckova L, Majerova K, et al. Comparative analysis of the visual performance after cataract surgery with implantation of a bifocal or trifocal diffractive IOL. J Refract Surg. 2014;30 (10):666-672. doi:10.3928/1081597X-20140903-06

5. Kretz FTA, Choi CY, Müller M, et al. Visual outcomes, patient satisfaction and spectacle independence with a trifocal diffractive intraocular lens. Korean J Ophthalmol. 2016;30(3):180-191. doi:10.3341/kjo.2016.30.3.180

6. Xu X, Zhu MM, Zou HD. Refractive versus diffractive multifocal intraocular lenses in cataract surgery: a meta-analysis of randomized controlled trials. J Refract Surg. 2014;30:634-644. doi:10.3928/ 1081597X-20140814-04

7. Davidson RS, Dhaliwal D, Hamilton DR, et al. ASCRS refractive cataract surgery subcommittee. Surgical correction of presbyopia. J Cataract Refract Surg. 2016;42:920-930. doi:10.1016/j.jcrs.2016.05.003

8. Pineda R, Chauhan T. Phakic intraocular lenses and their special indications. J Ophthalmic Vis Res. 2016;11(4):422-428. doi:10.410 3/2008-322X.194140

9. Kohnen T. Phakic intraocular lenses: where are we now? J Cataract Refract Surg. 2018;44(2):121-123. doi:10.1016/j.jcrs.2018.03.005

10. Barsam A, Allan BD. Excimer laser refractive surgery versus phakic intraocular lenses for the correction of moderate to high myopia. Cochrane Database Syst Rev. 2014. 6. doi:10.1002/14651858. CD007679.pub4

11. Tahzib NG, Nuijts RM, Wu WY, et al. Long-term study of artisan phakic intraocular lens implantation for the correction of moderate to high myopia: ten-year follow-up results. Ophthalmology. 2007;114 (6):1133-1142. doi:10.1016/j.ophtha.2006.09.029

12. Kohnen T, Shajari M. Phake Intraokularlinsen (phakic intraocular lenses). Ophthalmologe. 2016;113(6):529-538. doi:10.1007/s00347016-0283-Z

13. Kohnen T, Kook D, Morral M, Guell JL. Phakic intraocular lenses. Part 2: results and complications. J Cataract Refract Surg. 2010;36 (12):2168-2194. doi:10.1016/j.jcrs.2010.10.007

14. Dick HB, Budo C, Malecaze F, et al. Foldable artiflex phakic intraocular lens for the correction of myopia; two-year follow-up results of a prospective European multicenter study. Ophthalmology. 2009;116 (4):671-677. doi:10.1016/j.ophtha.2008.12.059

15. Chang DH, Davis EA. Phakic intraocular lenses. Curr Opin Ophthalmol. 2006;17(1):99-104. doi:10.1097/01.cco.0000188624.54743.c7

16. Shimizu K, Kamiya K, Igarashi A, et al. Long-term comparison of posterior chamber phakic intraocular lens with and without a central hole (hole ICL and conventional ICL) implantation for moderate to high myopia and myopic astigmatism. Medicine. 2016;95(14):e3270. doi:10.1097/MD.0000000000003270

17. Gomez-Bastar A, Jaimes M, Graue-Hernandez EO, et al. Long-term refractive outcomes of posterior chamber phakic (spheric and toric implantable collamer lens) intraocular lens implantation. Int Ophthalmol. 2014;34 (3):583-590. doi:10.1007/s10792-013-9860-1

18. Pérez-Vives C, Ferrer-Blasco T, Cerviño-Expósito A, et al. Simulated prototype of posterior chamber phakic intraocular lens for presbyopia correction. J Cataract Refract Surg. 2015;41(10):2266-2273. doi:10.1016/j.jcrs.2015.10.050

19. Vasavada V, Srivastava S, Vasavada SA, et al. Safety and efficacy of a new phakic posterior chamber IOL for correction of Myopia: 3 years of follow-up. $J$ Refract Surg. 2018;34(12):817-823. doi:10.3928/1081597X-20181105-01

20. Sachdev G, Ramamurthy D. Long-term safety of posterior chamber implantable phakic contact lens for the correction of myopia. Clin Ophthalmol. 2019;13:137-142. doi:10.2147/OPTH.S185304

21. Mehrjerdi MZ, Mohebbi M, Zandian M. Review of static approaches to surgical correction of presbyopia. J Ophthalmic Vis Res. 2017;12 (4):413-418. doi:10.4103/jovr.jovr_162_16 


\section{Publish your work in this journal}

Clinical Ophthalmology is an international, peer-reviewed journal covering all subspecialties within ophthalmology. Key topics include: Optometry; Visual science; Pharmacology and drug therapy in eye diseases; Basic Sciences; Primary and Secondary eye care; Patient Safety and Quality of Care Improvements. This journal is indexed on PubMed

Submit your manuscript here: https://www.dovepress.com/clinical-ophthalmology-journal
Central and CAS, and is the official journal of The Society of Clinical Ophthalmology (SCO). The manuscript management system is completely online and includes a very quick and fair peer-review system, which is all easy to use. Visit http://www.dovepress.com/ testimonials.php to read real quotes from published authors. 